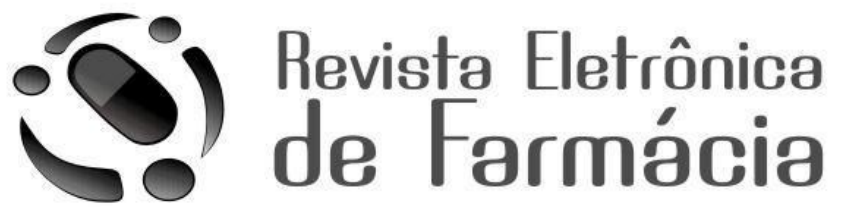

REF - ISSN 1808-0804 Vol. X (3), 11 - 19, 2013.

\title{
PERFIL DOS BETAS BLOQUEADORES ATENOLOL E ENALAPRIL DISPENSADOS EM FARMÁCIA MAGISTRAL DA CIDADE DE PELOTAS, RIO GRANDE DO SUL
}

PROFILE OF ATENOLOL AND BLOCKING BETAS ENALAPRIL EXEMPT IN THE CITY OF PHARMACY MAGISTRAL PELOTAS, RIO GRANDE DO SUL

PERFIL DE ATENOLOL Y BETAS BLOQUEO ENALAPRIL EXENTO EN LA CIUDAD DE FARMACIA MAGISTRAL PELOTAS, RIO GRANDE DO SUL

\section{${ }^{1}$ Rafael da Fonseca Prietsch}

${ }^{1}$ Mestrando pela Universidade Federal de Pelotas. Pelotas, Rio Grande do Sul.

Recebido em 08/08/2012, Aceito em 08/07/2013

\begin{abstract}
Resumo
O objetivo deste estudo, quantitativo, descritivo e retrospectivo, nos meses de 01/05/11 e 01/10/11 foi avaliar segundo a sua classe farmacológica, observar qual destes medicamentos anti-hipertensivos possui uma maior prevalência na utilização. O enalapril quando comparado a ao atenolol. O enalapril foi observado a dosagem de $5 \mathrm{mg}, 10 \mathrm{mg}$ e $20 \mathrm{mg}$ enquanto o atenolol foi de $25 \mathrm{mg}$ e $50 \mathrm{mg}$. Na distribuição dos anti-hipertensivos, segundo a sua classe farmacológica, observamos uma maior prevalência na utilização de enalapril quando comparado a ao atenolol.
\end{abstract}

Palavras-chave: Enalapril. Atenolol. Anti-hipertensivo e beta-bloqueadores. 
PRIETSCH, R. F. Revista Eletrônica de Farmácia Vol. X (3), 11 - 19, 2013.

\section{Abstract}

The objective of this study, quantitative, descriptive and retrospective, in the months of 01/05/11 and 01/10/11 was assessed according to its pharmacological class, seeing which antihypertensive drugs have a higher prevalence of use. Enalapril compared to atenolol. Enalapril was observed at doses of $5 \mathrm{mg}, 10 \mathrm{mg}$ and $20 \mathrm{mg}$ while atenolol was 25 $\mathrm{mg}$ and $50 \mathrm{mg}$. In the distribution of anti-hypertensive, according to its pharmacological class, we observed a higher prevalence in the use of enalapril compared to atenolol.

Uniterms: Enalapril. Atenolol. Anti-hypertensive and beta-blocker.

\section{Resumen}

El objetivo de este estudio, descriptivo, retrospectivo, en los meses de 01/05/11 y 01/10/11 fue evaluar en función de su clase farmacológica, observe cuál de estos fármacos antihipertensivos tienen una mayor prevalencia de uso. El enalapril en comparación con el atenolol. El enalapril se observó a dosis de $5 \mathrm{mg}, 10 \mathrm{mg}$ y $20 \mathrm{mg}$ de atenolol, mientras que era de $25 \mathrm{mg}$ y $50 \mathrm{mg}$. En la distribución de anticuerpos antihipertensiva, en función de su clase farmacológica, nos encontramos con una mayor prevalencia en el uso de enalapril en comparación con atenolol.

Palabras clave: Enalapril. Atenolol. Antihipertensivo y bloqueadores beta.

\section{INTRODUÇÃO}

A hipertensão é a doença cardiovascular mais comum que se tem conhecimento hoje em dia e está associada à pressão arterial elevada. No Brasil, em 2003, 27,4\% dos óbitos registrados foram decorrentes de doenças cardiovasculares ${ }^{(1)}$.

A mortalidade por doença cardiovascular aumenta progressivamente com a elevação da pressão arterial, a partir de 115/75 $\mathrm{mmHg}$ (SBH, 2006); muito embora, o risco de doença cardiovascular em pacientes com hipertensão seja determinado não apenas pelo nível da pressão arterial, mas também pela presença ou ausência de doença no órgão alvo ou fatores de risco, tais como tabagismo, dislipidemia e diabetes. A obesidade e a inatividade física também são preditores de risco cardiovascular ${ }^{(2-}$ 3).

A hipertensão arterial, quando persistente, danifica os vasos sangüíneos renais, cardíacos e cerebrais e resultam em aumento na incidência de insuficiência renal, cardíaca, de acidente vascular cerebral (AVC), infarto do miocárdio, etc. ${ }^{(4)}$. A avaliação inicial da hipertensão deve enfocar três objetivos $^{(4)}$ : 1- monitoramento da pressão arterial; 2- avaliação do risco cardiovascular global do paciente e 3detecção de pistas evidenciando causas potencialmente identificáveis de 
PRIETSCH, R. F. Revista Eletrônica de Farmácia Vol. X (3), 11 - 19, 2013.

hipertensão. Os dados clínicos iniciais necessários para atingir esses objetivos são obtidos através de uma história e um exame clínico meticuloso, exames de sangue e de urina e um eletrocardiograma. Em alguns pacientes, a monitoração ambulatorial da pressão arterial e um ecocardiograma fornecem dados adicionais úteis sobre o ônus tempo-integral da pressão arterial sobre o sistema cardiovascular ${ }^{(4)}$.

Um conceito reafirmado pelo Joint National Committee (JNC) de Prevenção, Deteç̧ão, Avaliação e Tratamento da pressão arterial é o de que o aumento do risco cardiovascular se inicia com níveis de pressão arterial considerado normal e aumenta de forma diretamente proporcional ao aumento da pressão arterial $^{(2)}$. Diante disto o JNC no seu sétimo relatório ${ }^{(5)}$ propôs uma classificação da pressão arterial com algumas diferenças quando comparada à classificação proposta pelo SBH na $V$ Diretrizes Brasileiras de Hipertensão, sendo estabelecido pelo JNC um estado de pré-hipertensão para Pressão Arterial Sistólica (PAS) entre 120-139 mmHg e Pressão Arterial Diastólica (PAD) de 80$89 \mathrm{mmHg}$. Ainda assim, é importante destacar que o limite de 140/90 mmHg continua como o ponto de corte para a identificação e caracterização de hipertensão em todos os documentos oficiais que tratam da hipertensão arterial $^{(6)}$.

O tratamento da hipertensão deve persistir por toda a vida, pelo fato de, até hoje, essa doença não ter cura. No entanto, geralmente pode ser controlada por modificações no estilo de vida do paciente e com o uso de medicamentos. O objetivo é a redução na pressão arterial e nas anormalidades metabólicas associadas para se reduzir o risco de doenças cardiovasculares e renais, sem comprometer a qualidade de vida do paciente ${ }^{(4)}$.

Como a terapia anti-hipertensiva geralmente não é dirigida para uma causa específica, ela, necessariamente, tem de interferir nos mecanismos fisiológicos normais que regulam a pressão arterial. A terapia antihipertensiva é administrada a um paciente assintomático, ao qual ela não proporciona alívio direto de qualquer desconforto. Com efeito, o benefício da redução da pressão arterial consiste em prevenir a ocorrência de doenças e a morte em algum momento do futuro. A tendência humana natural de atribuir maior peso a uma inconveniência e ao desconforto presentes do que a qualquer benefício futuro possível representa um importante problema na terapia antihipertensiva, que é oferecer uma terapia farmacológica eficaz e consistente durante muitos anos num esquema que o paciente deverá seguir( ${ }^{(7)}$.

O tratamento farmacológico da hipertensão é feito com o uso de agentes anti-hipertensivos. Essas drogas atuam nos mecanismos que interferem a regulação da pressão arterial. Uma classificação útil desses fármacos 
PRIETSCH, R. F. Revista Eletrônica de Farmácia Vol. X (3), 11 - 19, 2013.

consiste em dividi-los de acordo com o principal local regulador ou mecanismo sobre o qual atuam ${ }^{(7,8)}$.

Os bloqueadores $\beta$ se subdividem em cardioseletivos e não cardioseletivos de acordo com a atividade bloqueadora $\beta-2$. Os bloqueadores $\beta$ atuam bloqueando os receptores $\beta$ encontrados em várias partes do corpo, incluindo coração, cérebro e vasos sanguíneos. Quando esses receptores presentes no coração são bloqueados, os batimentos cardíacos diminuem e o coração bate com menos força, reduzindo a pressão sangüínea. Eles também reduzem a energia usada pelo coração para bombear o sangue pelo organismo, reduzindo assim a necessidade de oxigênio por esse órgão ${ }^{(9)}$.

$O$ atenolol é um fármaco que pertence ao grupo dos bloqueadores $\beta$ caridoseletivos e pode ser usado no tratamento anti-hipertensivo por reduzir a pressão sangüínea, no tratamento contra a angina ("dor no peito") por aliviar seus sintomas, como tratamento da arritmia por regular os batimentos cardíacos e como tratamento de infarto do miocárdio(10).

Quimicamente 0 atenolol pode ser descrito como (RS)-4-(2-hidroxi-3isopropilaminopropoxi) fenilacetamida. É um composto com massa molar de aproximadamente 266, relativamente polar, hidrofílico e com carga positiva em $\mathrm{pH}^{\prime}$ s acima do $\mathrm{pH}$ fisiológico, $\pm 7,3^{(11)}$.

A absorção do atenolol após a administração oral é consistente, mas incompleta (aproximadamente 40-50\%), com picos de concentração plasmática que ocorrem 2-4 horas após a administração da dose. Os níveis sanguíneos de atenolol são consistentes e sujeitos à pequena variabilidade. Não há metabolismo hepático significativo e mais de $90 \%$ da quantidade absorvida alcançam a circulação sistêmica inalterada. A meia-vida plasmática é cerca de 6 horas, mas pode se elevar na presença de comprometimento renal grave, uma vez que os rins são a principal via de eliminação(12).

Pó cristalino Branco. Ligeiramente solúvel em água e álcool e com ponto de fusão entre $152-156,5^{\circ} \mathrm{C}$.

Em virtude de seu mecanismo de ação comum, as drogas antihipertensivas, incluindo o atenolol, tendem a produzir um espectro semelhante de toxicidade, causando efeitos colaterais tais como diarréia, náusea, depressão, cansaço, colite isquêmica, trombose, etc. ${ }^{(13)}$, principalmente se for administrada de forma oral.

O uso de matrizes poliméricas para a liberação controlada do atenolol. A primeira propôs $o$ uso do hidroxipropilmetilcelulose, enquanto o segundo propôs a otimização de um dispositivo de liberação composto pelo Carbopol®, usando a metodologia de resposta de superfície. Ambos os trabalhos obtiveram resultados positivos quanto ao objetivo e quanto à metodologia usada para a proposta 
PRIETSCH, R. F. Revista Eletrônica de Farmácia Vol. X (3), 11 - 19, 2013.

sugerida $^{(14,15)}$.

O maleato de enalapril é derivado peptídico sintético. Corresponde ao éster etílico da 1-[[carboxi-3-fenilpropil]-Lalanil-L-prolina] (Gomez et al, 1985; Kelly et al, 1986; Edeki et al, 1993). É uma pró-droga qua após sua absorção é hidrolisado no fígado em sua forma ativa Enalaprilato (MK-22), um potente inibidor da ECA (Lowewnthal, 1985; Frishman, 1990 e Gross, 1980).

A fórmula molecular é C2OH28N2O5.C4H4O4, a massa molecular é 429,53 g.mol-1.

O maleato enalapril (MK-21), após sua administração oral, apresenta boa absorção (60 a 70\%) pelo trato

\section{MATERIAL E MÉTODOS}

\section{Caracterização do local de estudo}

A cidade de Pelotas está localizada a encosta sudeste, ás margens do canal São Gonçalo e distante da capital do estado, Porto Alegre, 250 KM. Possui uma área de $1.921 .80 \mathrm{Km}^{2}$. A cidade tem uma população de 323.034 habitantes, sendo Homens 153.180 habitantes e Mulheres 169.854 habitantes. Temperatura anual de $17,6^{\circ} \mathrm{C}$. Seu clima e subtropical úmido. A altitude média é de 7 metros acima do nível do mar.

\section{Levantamento dos dados}

Realizou-se um estudo quantitativo, descritivo e retrospectivo, onde foram avaliadas as seguintes concentrações de atenolol, $25 \mathrm{mg}$ e $50 \mathrm{mg}$ gastrointestinal (Todd \& Goa, 1992 e Ulm, 1983). O pico de concentração plasmática máxima (Cmax) é alcançado cerca de 1 hora após a administração da droga (Todd \& Goa, 1992; Todd \& Heel, 1986 e UIm, 1993).

Trata-se de um pó cristalino de cor de creme de leite ou de uma coloração tipo branco "sujo". É livremente solúvel em álcool etílico, álcool metílico e dimetilformamida; ligeiramente solúvel em água. É rapidamente absorvido e hidrolisado a enalaprilato, inibidor da angiotensina de ação prolongada.

e de enalapril $5 \mathrm{mg}, 10 \mathrm{mg}$ e $20 \mathrm{mg}$ num período de 6 meses $(01 / 05 / 2011$ a 01/10/2011).

\section{Resultados e discussão}

$\mathrm{Na}$ distribuição dos antihipertensivos nos meses de 01/05/2011 a 01/10/2011, segundo a sua classe farmacológica, observamos uma maior prevalência na utilização de enalapril quando comparado a ao atenolol. 
PRIETSCH, R. F. Revista Eletrônica de Farmácia Vol. X (3), 11 - 19, 2013.

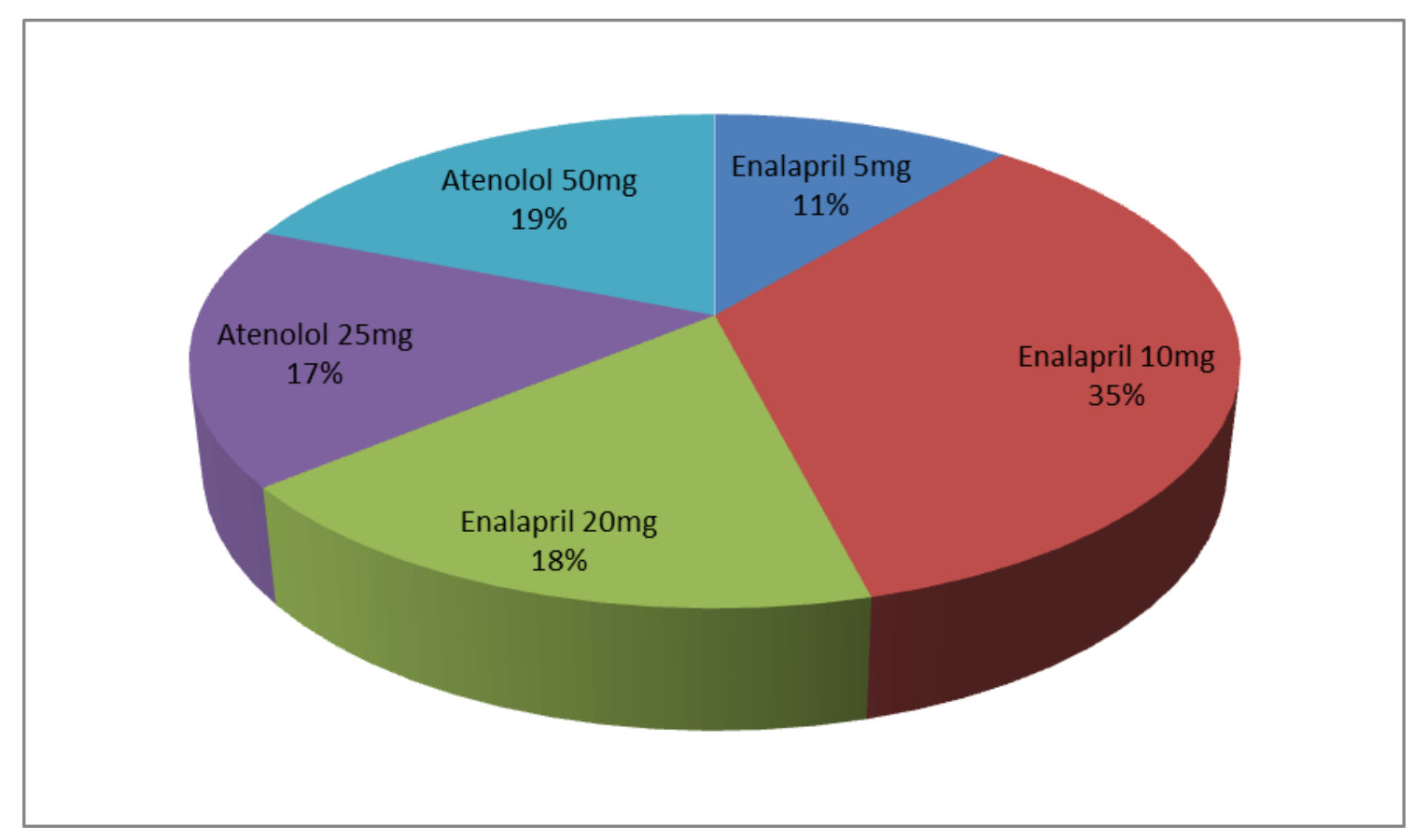

Os resultados mostram que no período estudado, de 01/05/2011 a 01/10/2011, as formulações à base de enalapril apresentara maior prevalência em comparação ao outro beta bloqueador. A predominância das formulações à base de enalapril pode ser explicada pelo fato dessa classe farmacológica apresentar 3 diferentes concentrações, enquanto 0 atenolol possui 2 concentrações usuais.
Como aspectos positivos do estudo: pode-se ressaltar o fato de ser o primeiro a retratar a consumo dos antihipertensivos na região Sul do município de Pelotas.

Abaixo gráfico em relação ao consumo de atenolol de $25 \mathrm{mg}$ comparado ao atenolol de $50 \mathrm{mg}$. 
PRIETSCH, R. F. Revista Eletrônica de Farmácia Vol. X (3), 11 - 19, 2013.

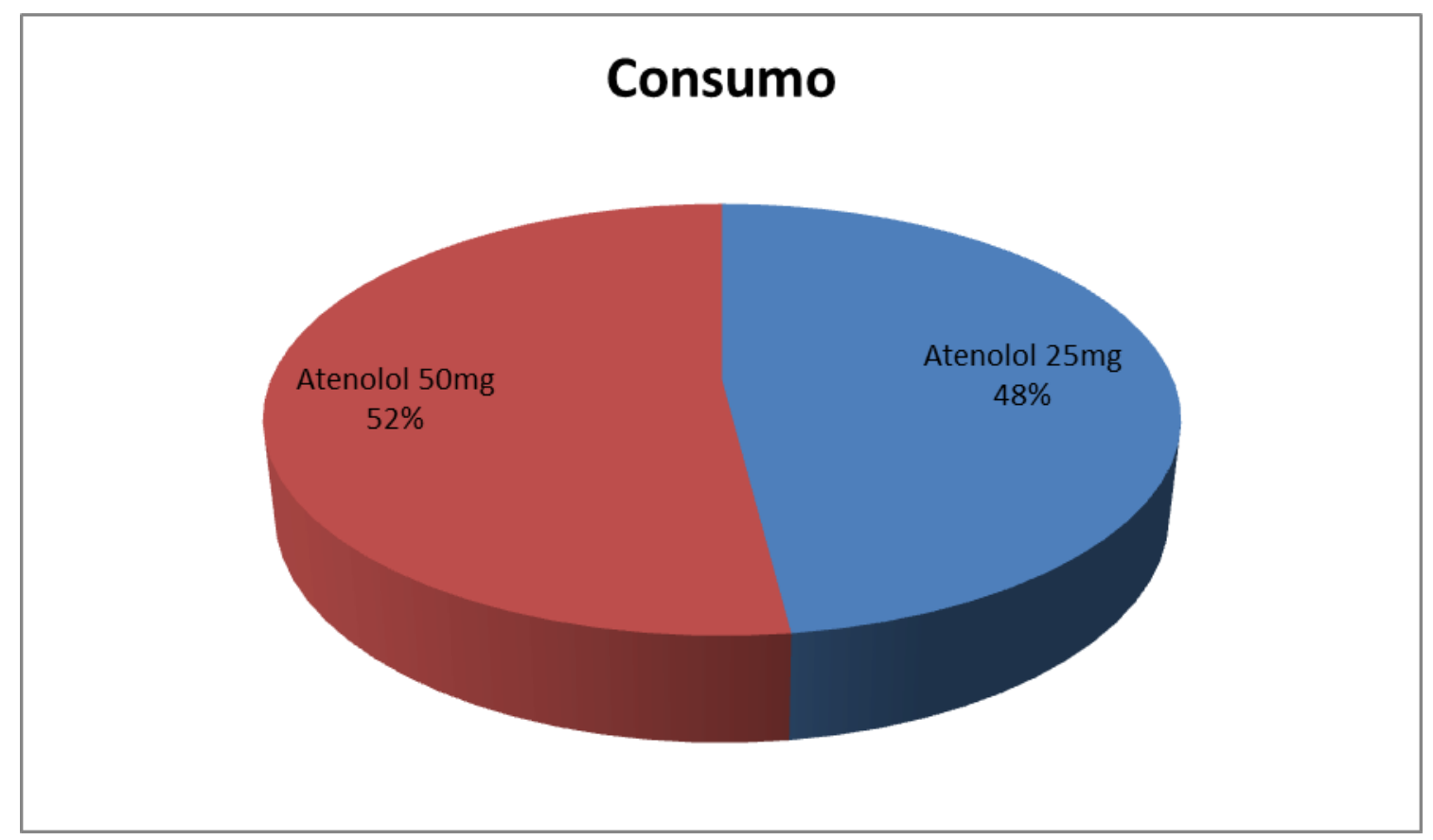

Observa-se uma maior prescrição dos atenolol 50mg quando este é comparado ao atenolol, $25 \mathrm{mg}$.

Abaixo gráfico em relação ao consumo de enalapril de $5 \mathrm{mg}$ comparado ao enalapril de $10 \mathrm{mg}$ e $20 \mathrm{mg}$.

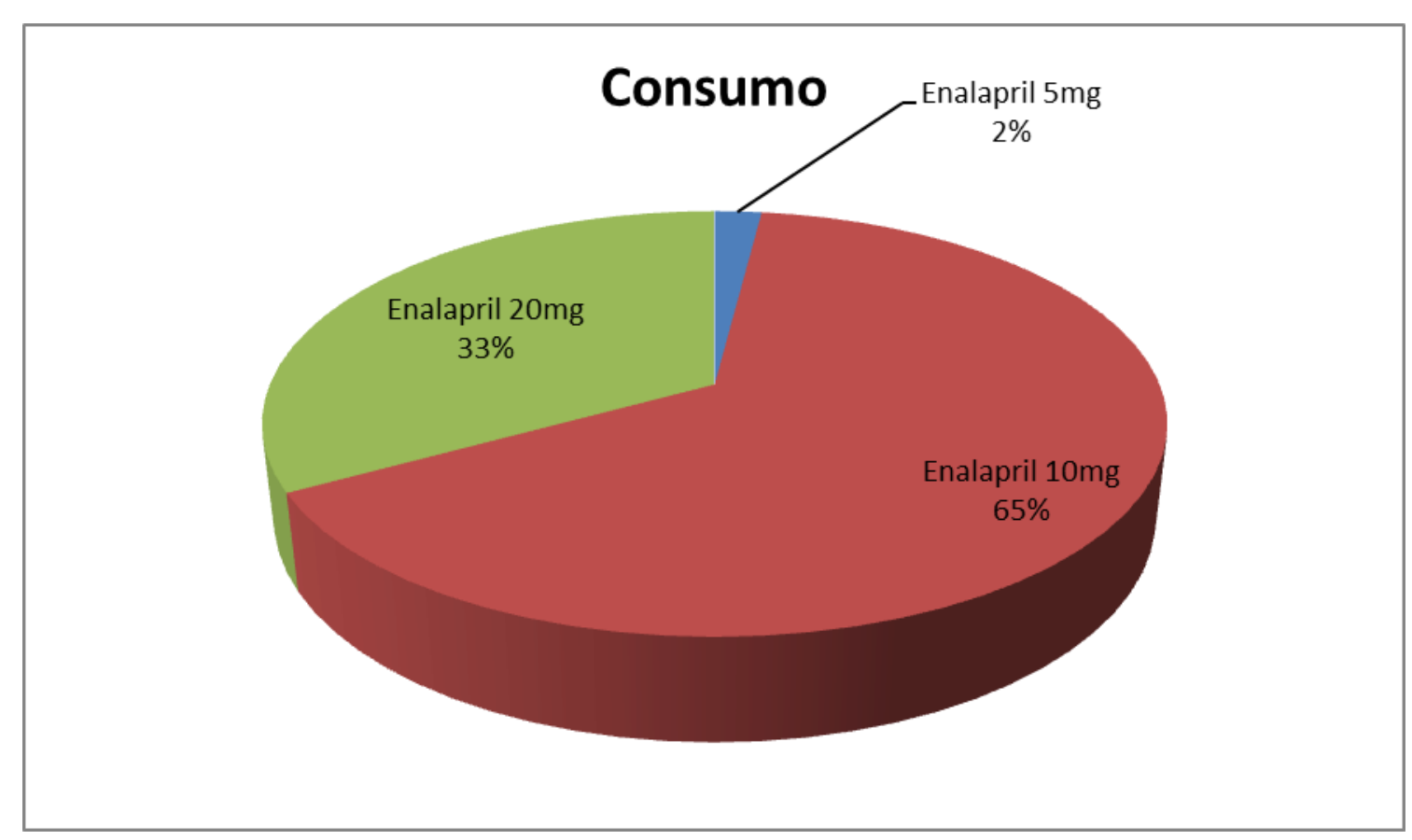

Observa-se uma maior prescrição comparado ao enalapril de $5 \mathrm{mg}$ e $20 \mathrm{mg}$. do enalapril $10 \mathrm{mg}$ quando este é CONCLUSÕES 
PRIETSCH, R. F. Revista Eletrônica de Farmácia Vol. X (3), 11 - 19, 2013.

Em geral o resultado obtido desse estudo para o município de Pelotas como um todo seria algo incorreto, uma vez que o presente trabalho analisou uma amostra reduzida para tais propósitos. Qualquer tipo de extrapolação poderá induzir a reprodução de dados e informações não consistentes. Contudo, estes resultados preliminares apresentados neste trabalho mostram que o consumo de enalapril na área estudada foi similar ao relatado em outros trabalhos.

\section{REFERÊNCIAS}

1. SBH (Sociedade Brasileira de Hipertensão). Hipertensão arterial. V Diretrizes Brasileira de Hipertensão, 2006.

2. NIH (National Institutes of Health). VI Relatório do Joint National Committee on Prevention, Detection, Evaluation, and Treatment of High Blood Presure, 1997.

3. Whelton PK. Hypertension curriculum review: epidemiology and the prevention of hypertension. Journal of Clinical Hypertension, 2004(6):636-642.

4. Goldman L, Ausiello D. CECIL - Tratado de Medicina Interna. 22. ed. Elsevier, 2005.

5. NIH (National Institutes of Health). The Seventh Report of the Joint National Committee on Prevention, Detection, Evaluation, and Treatment of High Blood Presure, 2003.

6. Pozzan R, Brandão AA, Magalhães ME, Freitas EV, Brandão AP. O controle da pressão arterial como questão central no tratamento da hipertensão arterial. Revista Brasileira de Hipertensão, 2003(10):253-259.

7. Katzung BG. Farmacologia - Básica \& Clínica. 9. ed. Guanabara Koogan, 2006.

8. Gilman AG. As Bases Farmacológicas da Terapêutica. Ed. McGraw-Hill, 9. ed. 1996.

9. <http://www.clinicalanswers.nhs.uk/index.cfm?question=2611>. Acesso em 02 set. 2011.

10. http://www.sunderland.ac.uk/ hsOdad/profile/atenolol/struc.htm. Acesso em 27 set. 2011

11. Jacobsen J. Buccal Iontophoretic Delivery of Atenolol. $\mathrm{HCl}$ Employing a new in vitro Three-Chamber Permeation Cell. Journal of Controlled Release, 2001(70):83-95.

12. <http://www.ccs.ufsc.br/farmacia/TCCGenericos/MecAtenolol.html>. Acesso em: 20 ago. 2011.

13. Cho CW, Shin SC. Enhanced Transdermal Delivery of Atenolol from the Ethylene-Vinyl Acetate Matrix. International Journal of Pharmaceutics, $2004 ;(287): 61-71$.

14. Vázquez MJ, Casalderrey M, Duro R, Gómez-Amonza JL, Martínez-Pacheco R, Souto C. et al. Atenolol Release from Hydrophilic Matrix Tablets with Hydroxypropylmethylcellulose (HPMC) mixtures as Gelling Agent: Effects of the Viscosity of the HPMC Mixture. European Journal of Pharmaceutical Sciences, 1996;(4):39-48. 
PRIETSCH, R. F. Revista Eletrônica de Farmácia Vol. X (3), 11 - 19, 2013.

15. Singh B, Chakkal SK, Ahuja N. Formulation and Optimization of Controlled Release Mucoadhesive Tablets of Atenolol Using Response Surface Methodology. AAPS PharmSciTech, 7(1), artigo 3, 2006. 